\title{
A Case of Severe Esophageal Intramural Pseudodiverticulosis Whose Symptoms Were Ameliorated by Oral Administration of Anti-Fungal Medicine
}

\author{
Takashi Chiba Katsunori lijima Tomoyuki Koike \\ Kaname Uno Naoki Asano Tooru Shimosegawa \\ Division of Gastroenterology, Tohoku University Graduate School of Medicine, \\ Sendai, Japan
}

\section{Key Words}

Anti-fungal therapy · Candida albicans · Endoscopic balloon dilatation · Esophageal intramural pseudodiverticulosis

\begin{abstract}
Esophageal intramural pseudodiverticulosis (EIPD) is a rare disease of unknown etiology that displays multiple pseudodiverticula radiologically, leading to benign esophageal stricture. Dysphagia, which sometimes slowly progresses, is the main symptom in the majority of cases. We here report a 59-year-old male EIPD patient who suffered from severe dysphagia. Radiography and endoscopy of this patient disclosed a severe constriction in the upper thoracic esophagus. Although we tried several endoscopic procedures including frequent endoscopic balloon dilatation (EBD), the effect was very limited and his dysphagia relapsed shortly after the treatments. During the procedures, we noticed some white, thick, creamy liquid emerging from the orifices of EIPD, and PAS staining of biopsy specimens revealed infection with Candida albicans. Hence, the patient was given anti-fungal medicine in addition to EBD. The additional treatment with anti-fungal medicine dramatically improved his symptoms and the esophageal constriction. This case suggests that anti-fungal treatment is an effective first-line therapy even against a severe form of esophageal constriction in EIPD.
\end{abstract}




\section{Introduction}

Esophageal intramural pseudodiverticulosis (EIPD) is a rare disease of unknown origin which was first described by Mendl in 1960 [1]. In the majority of cases dysphagia, which sometimes slowly progresses, is the main symptom [2, 3]. EIPD is often associated with gastroesophageal reflux, chronic alcohol abuse, diabetes and Candida infection, but its etiology and pathogenesis remain obscure [2, 3]. It often complicates severe stricture, which makes double contrast esophagogram and computed tomography more useful than esophagogastroduodenoscopy (EGD) in diagnosing EIPD [2]. The former two procedures reveal multiple, small, flask-shaped outpouchings in the wall of the esophagus, which are the characteristics of EIPD. Almost all cases of EIPD are benign and responds well to medical treatment for inflammation and to endoscopic dilatation of the esophagus [2,3]

We here report a suggestive case of a severe form of EIPD whose dysphagia was ameliorated not by endoscopic dilatation therapy but by oral administration of anti-fungal medicine.

\section{Case Report}

A 59-year-old man suffered from dysphagia since 1995, but he did not consult any hospital until the symptom worsened in 2008. When he consulted a nearby hospital, EGD revealed severe constriction in the thoracic esophagus, and he was referred to our hospital for treatment. He had undergone subtotal gastrectomy with Billroth II reconstruction for gastric ulcer when 40 years old. Otherwise he had no remarkable past history. Physical examination showed no unusual findings, but laboratory data showed anemia ( $\mathrm{Hb}: 10.1 \mathrm{~g} / \mathrm{dl}$ ), low serum albumin $(3.5 \mathrm{~g} / \mathrm{dl})$ and slight increase of tumor marker SCC (1.9 ng/ml; normal limit 0-1.5 ng/ml).

Radiography of the esophagus disclosed severe constriction in the upper thoracic esophagus. The esophageal lumen was slightly dilated on the oral side of the narrowing, where several sac-like barium collections (up to 1-2 cm in diameter) protruded. The barium barely thread through a 5- to 6-cm-long segment of severe narrowing, and the anal side of the narrowing only slightly appeared on the radiography (fig. 1a). Esophagoscopy revealed a severe, fibrotic stenosis $26 \mathrm{~cm}$ from the incisors. On the oral side of the stenosis, the esophageal mucosa was occupied by honeycomb-like multiple depressions (fig. 2a, b). A thin-caliber endoscope (6-mm outside diameter; Olympus XP260N) barely passed through this narrowed region where the esophageal mucosa was not ulcerated (fig. $2 \mathrm{c}$ ). Then a large number of tiny orifices corresponding to pseudodiverticula were identified along the anal side of the stenotic region (fig. 2d). Biopsies from the orifices and stenotic region detected only inflammatory changes and no malignancies. Computed tomography revealed wall thickening and small gas collection, which corresponded to pseudodiverticula, in the wall of the upper to middle thoracic esophagus.

As endoscopic examination revealed a severe fibrotic stenosis, endoscopic mechanical dilatation therapy was applied to this patient rather than medical treatment in expectation of prompt resolution of symptoms. We repeatedly performed endoscopic balloon dilatation (EBD) for the esophageal constriction, but the effect was very limited, and the dysphagia relapsed with esophageal re-stenosis shortly after the treatments. We then tried making an incision in the esophageal mucosa endoscopically using an IT knife [4]. This treatment improved his symptom and radiography also showed improvement of the constriction, but the dysphagia gradually worsened, and 6 months after the treatment, EGD revealed re-stenosis of the esophagus. He showed progressed malnutrition due to severe dysphagia and had lost $5 \mathrm{~kg}$ of body weight during 6 months.

Since a white, thick, creamy liquid suggestive of pus emerging from the orifices was sometimes detected endoscopically (fig. $3 \mathrm{a}, \mathrm{b}$ ) and Candida albicans was detected in PAS staining of the biopsy specimen, we began giving this patient an anti-fungal medicine in addition to performing EBD 
therapy, and these treatments greatly improved his dysphagia. EGD revealed significant improvement of the esophageal constriction and disappearance of the pus, although multiple small depressions remained in the entire esophagus (fig. 3c, d). Radiography showed significant improvement in the flow of swallowed barium, although there still remained a clumped filling defect in the upper thoracic esophagus (fig. 1b). It was also revealed by repeated radiography that very small (1-3 mm), multiple, flask-shaped outpouchings were diffusely distributed in the esophagus on the anal side of the narrowing (fig. 1c). The patient required continuous anti-fungal medicine because his symptom quickly worsened after temporary discontinuation of the medication. It is now 24 months since we began anti-fungal treatment, and he is still symptom-free with no severe constriction detected on EGD.

\section{Discussion}

EIPD is characterized by the development of multiple, epithelium-lined cysts within the esophageal wall; each cyst connects to the esophageal lumen via a narrow ostium. The cysts connected to the esophageal lumen result from ductal dilatation of the esophageal submucosal glands and excretory ducts, displaying multiple pseudodiverticula radiologically $[2,3]$. In the present case, characteristic, multiple, tiny, flask-shaped outpouchings were identified in the esophagus along the anal side of the narrowing. On the other hand, the esophageal mucosa on the oral side of the narrowing showed greater barium collection as occurs in fistula formation, which is somewhat unusual for EIPD and could have resulted from the branching or bridging between adjacent diverticula [2]. The long period before receiving medical care and concomitant Candida infection could be responsible for the unusual shape of EIPD in this case.

Since the first case report of EIPD in 1960 [1], there have numerous case reports and reviews concerning this rare esophageal disorder [2, 3, 5], but the present case could represent a most advanced condition of EIPD, considering that the swallowed barium barely thread through the stricture and esophagoscopy revealed a stiff, fibrotic stenosis. Previous reports documented that, while oral administration of medication is employed for patients with EIPD accompanied by esophagitis or esophageal candidiasis, EBD has been carried out for patients with severe constriction [2, 3]. Our case was resistant to endoscopic mechanical dilatation therapy, although previous reports consistently described that endoscopic dilatation of the stricture usually gives immediate and long-lasting relief of dysphagia in EIPD [2, 3, 6, 7]. In addition, this was the first attempt to treat severe stenosis of EIPD with endoscopic incision therapy, which has been successfully applied for the dilatation of benign strictures of the esophagus due to other causes [8], but this therapy also showed only a limited effect for resolution of the dysphagia in our case.

Remarkably, in spite of failures with endoscopic mechanic dilatation treatments for the severe stricture, oral administration of anti-fungal medication turned out to be effective in the treatment of EIPD, leading to long-lasting resolution of the dysphagia in the present case. Re-examination by radiography and EGD during the anti-fungal medication showed that the constriction of the esophagus had also improved, although the tiny diverticula did not resolve after the treatment. Subsequently, the patient required continuous anti-fungal medication for sustained improvement of the dysphagia. 
There are two possible causes of ductal dilatation in EIPD. The first is an obstruction of the ducts due to inflammatory cells or desquamated epithelium, and the second is compression of the ductal orifices due to periductal infiltrates and/or fibrosis $[2,3,9]$. Previous reports showed that $C$. albicans infection is frequently present in EIPD, and such infection may be involved in the etiology of EIPD in either mechanism [2,3]. The clinical course of our case also suggests a significant contribution of Candida infection in the exacerbation of the EIPD. In the present case, in addition to pre-existing chronic fibrosis of the esophageal wall, concomitant inflammatory cellular infiltration triggered by Candida infection may have worsened the stricture of EIPD, possibly by enhanced intramural pressure in the narrowed esophagus [5].

In conclusion, we experienced a case of an advanced stage of esophageal stricture derived from EIPD, which was resistant to endoscopic mechanical dilatation therapy. Even in such a severe case, anti-fungal medication was still effective in relieving the persistent dysphagia. An important lesson of this case is that anti-fungal medication, rather than endoscopic dilatation therapy, could be considered as a first-line therapy against dysphagia and constriction in EIPD regardless of the severity of the esophageal constriction. 

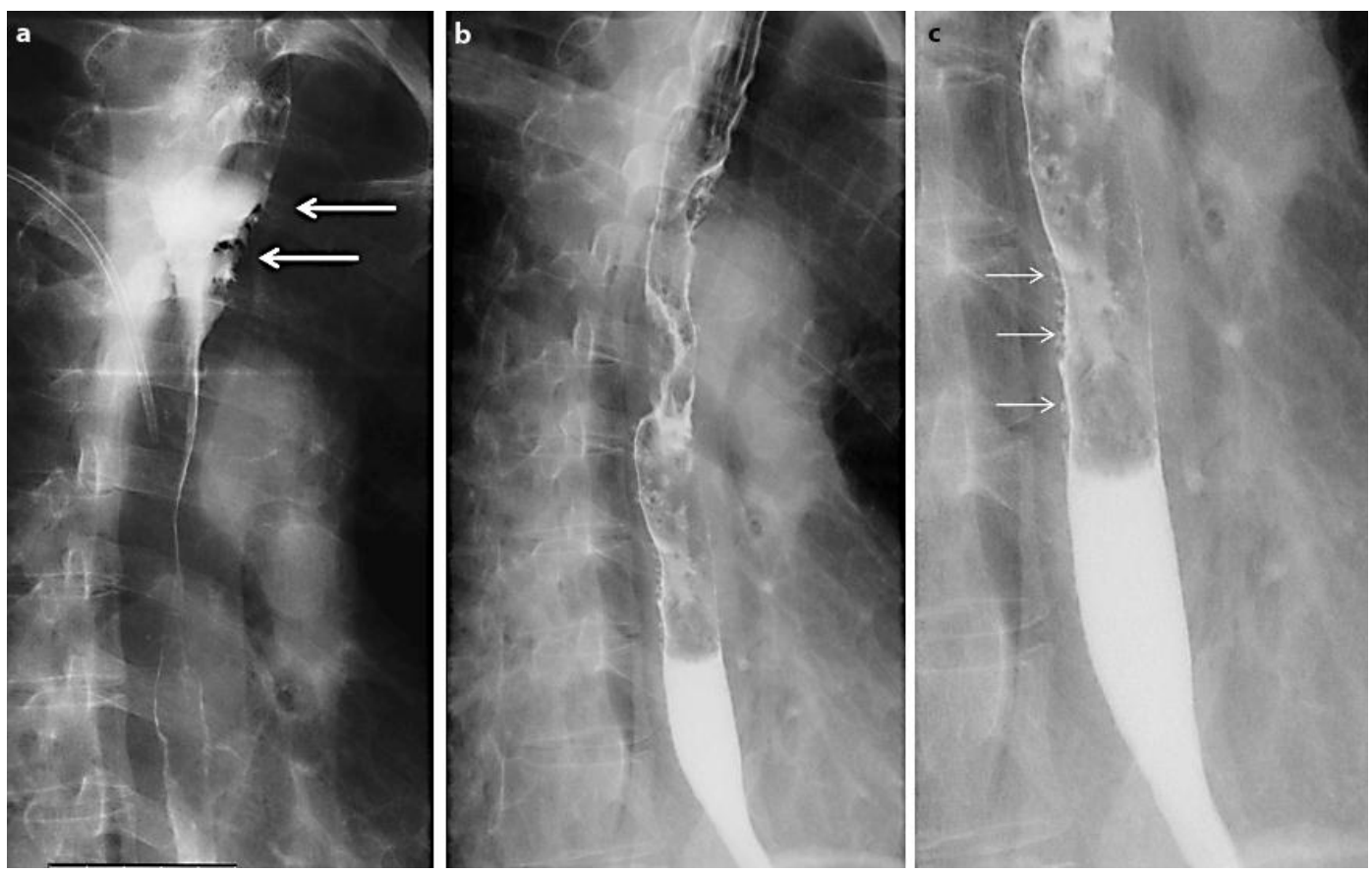

Fig. 1. Radiography of the esophagus before and after treatment. a Before any treatment, the esophagus was severely stenosed in the upper thoracic area. Arrows represent protruding sac-like barium collections. b Radiography of the esophagus 3 months after commencement of oral administration of anti-fungal medicine indicated that the flow of the swallowed barium was greatly improved during the administration of anti-fungal medicine, although there still remained a clumped filling defect in the upper thoracic esophagus. c On the anal side of the narrowing, very small (1-3 mm) multiple flask-shaped outpouchings (arrows), representing multiple pseudodiverticula, were diffusely distributed in the esophagus. 

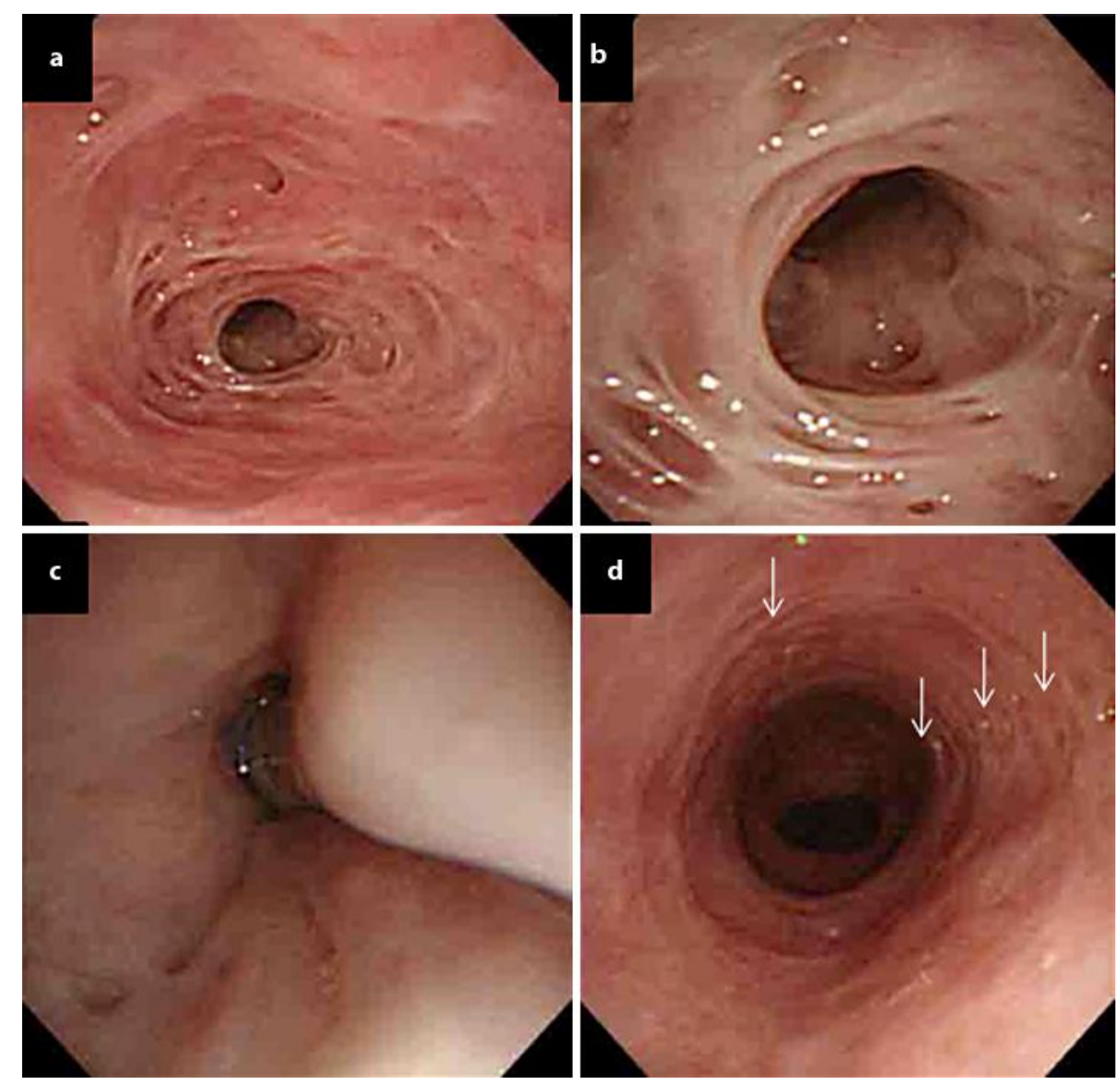

Fig. 2. Endoscopic images of the esophagus before treatment. a, b On the oral side of the stenosis, the esophageal mucosa was occupied by honeycomb-like multiple depressions. c Severe fibrotic stenosis was observed $26 \mathrm{~cm}$ from the incisors. $d$ Along the anal side of the stenotic region, large numbers of tiny orifices (arrows) were distributed. 

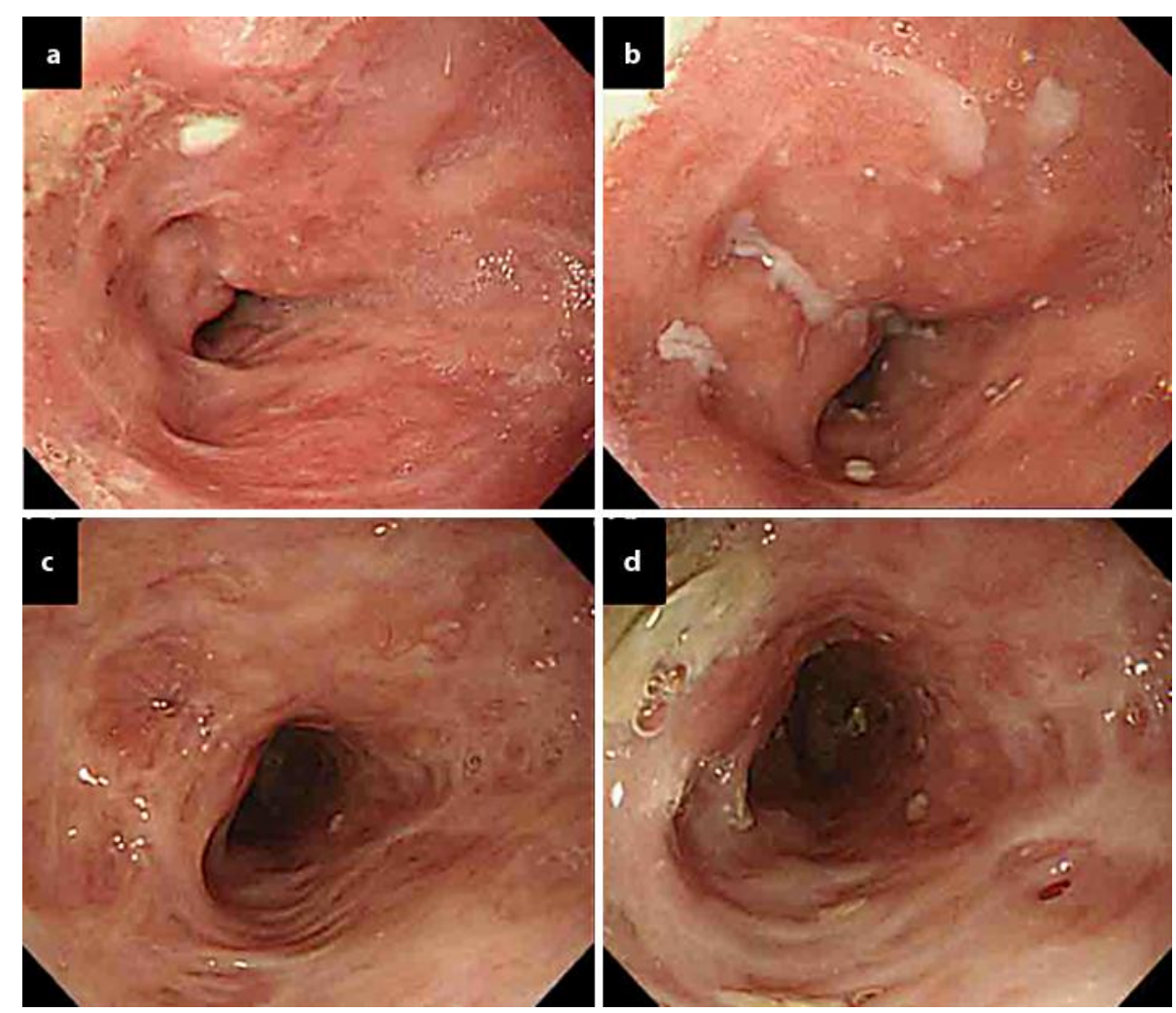

Fig. 3. Endoscopic images before and after continuous oral administration of anti-fungal medicine. a, b Prior to the anti-fungal medicine, the esophageal lumen was severely stenosed, and a white, thick, creamy liquid suggestive of pus emerging from the orifices was detected endoscopically. c, $\mathbf{d}$ Three months after commencement of the treatment, the esophageal stenosis was considerably improved and the pus from the orifices had disappeared.

\section{References}

1 Mendl K, McKay JM, Tanner CM: Intramural diverticulosis of the esophagus and Rokitansky-Aschoff sinuses in the gallbladder. Br J Radiol 1960;33:496-501.

-2 Brühlmann WF, Zollikofer CL, Maranta E, Hefti ML, Bivetti J, Giger M, Wellauer J, Blum AL: Intramural pseudodiverticulosis of the esophagus: report of seven cases and lite rature review. Gastrointest Radiol 1981;6:199-208.

-3 Umlas J, Sakhuja R: The pathology of esophageal intramural pseudodiverticulosis. Am J Clin Pathol 1976;65:314-320.

4 Ono H: Endoscopic submucosal dissection for early gastric cancer. Chin J Dig Dis 2005;6:119-121.

5 Koyama S, Watanabe M, Iijima T: Esophageal intramural pseudodiverticulosis (diffuse type). J Gastroenterol 2002;37:644-648.

6 Yoneyama F, Kobayashi Y, Miyata K, Ohta H, Takeuchi E, Yamada T, Hattori T: Esophageal intramural pseudodiverticulosis treated by balloon dilatation: report of a case. Surg Today 2004;34:62 -64. 
-7 Teraishi F, Fujiwara T, Jikuhara A, Kamitani S, Morino Y, Sato K, Tanaka N: Esophageal intramural pseudodiverticulosis with esophageal strictures successfully treated with dilation therapy. Ann Thorac Surg 2006;82:1119-1121.

$\checkmark 8$ Hordijk ML, Siersema PD, Tilanus HW, Kuipers EJ: Electrocautery therapy for refractory anastomotic strictures of the esophagus. Gastrointest Endosc 2006;63:157-163.

$>9$ Medeiros LJ, Doos WG, Balogh K: Esophageal intramural pseudodiverticulosis: a report of two cases with analysis of similar, less extensive changes in 'normal' autopsy esophagi. Hum Pathol 1988;19:928-931. 